\title{
A new area-based incumbent protection technique based on three-dimensional beamforming for dynamic spectrum access in a millimeter-wave band
}

\author{
Keita Onose $^{1, \text { a) }}$, Hiroto Kuriki ${ }^{1}$, Ryota Kimura ${ }^{1}$, and Ryo Sawai ${ }^{1}$ \\ ${ }^{1}$ Sony Corporation, 1-7-1 Konan Minato-ku, Tokyo, 108-0075 Japan \\ a)Keita.Onose@sony.com
}

Abstract: In this letter, we propose an advanced incumbent protection method that considers three-dimensional aspects of 5G New Radio (NR) beamforming for dynamic spectrum access (DSA). The proposed method further introduces a concept of area-based protection to satisfy protection criteria for a primary system (PS) with inaccurate location information. We show some computer simulation results for deploying a $5 \mathrm{G}$ based secondary system (SS) in a millimeter-wave band which is primarily assigned to a fixed wireless access (FWA) based PS. The results demonstrate that the proposed method increases allowable transmission powers of the SS by up to $15 \mathrm{~dB}$, compared to a conventional method.

Keywords: dynamic spectrum access, incumbent protection, spectrum management/access system, beamforming, transmission power control

Classification: Wireless Communication Technologies

\section{References}

[1] M.M. Sohul, M. Yao, T. Yang, and J.H. Reed, "Spectrum access system for the citizen broadband radio service," IEEE Commun. Mag., vol. 53, no. 7, pp. 18-25, July 2015. DOI: 10.1109/MCOM.2015.7158261

[2] "Implementing TV white spaces," The Office of Communications (Ofcom), London, United Kingdom, https://www.ofcom.org.uk/_data/assets/pdf_file/ 0034/68668/tvws-statement.pdf, Feb. 2015.

[3] "ECC report 205 licensed shared access (LSA)," European Conference of Postal and Telecommunications Administrations (CEPT), Copenhagen, Denmark, http://spectrum.welter.fr/international/cept/ecc-reports/ecc-report-205LSA-2300-MHz-2400-MHz.pdf, Feb. 2014.

[4] "Frequency reorganization action plan (FY2020 second revision)," Ministry of Internal Affairs and Communications (MIC), Tokyo, Japan, https://www. soumu.go.jp/main_content/000704672.pdf, Nov. 2020 (in Japanese).

[5] H. Kuriki, K. Onose, R. Kimura, and R. Sawai, "Area-based primary protection with antenna rotation prediction for dynamic spectrum access," Proc. 2020 International Conference on Emerging Technologies for Communications, B3-4, pp. $1-4$, Dec. 2020 
[6] Wireless Innovation Forum, "Requirements for commercial operation in the U.S. 3550-3700 MHz citizens broadband radio service band," WINNF-TS-0112 version V1.9.0, Dec. 2019.

[7] ITU Radiocommunication Sector, "Liaison statement to Task Group 5/1 - Spectrum needs and [characteristics for the terrestrial component of IMT in the frequency range between $24.25 \mathrm{GHz}$ and $86 \mathrm{GHz}$," Feb. 2017.

[8] 3GPP, "TR 38.901 Study on channel model for frequencies from 0.5 to 100 GHz,”Jan. 2020.

[9] ITU Radiocommunication Sector, "Reference radiation patterns for fixed wireless system antennas for use in coordination studies and interference assessment in the frequency range from $100 \mathrm{MHz}$ to $86 \mathrm{GHz}$," ITU-R Recommendation F699-8, Jan. 2018.

\section{Introduction}

Dynamic spectrum access (DSA) technology has attracted much attention as one of core technologies to solve a spectrum/frequency resource shortage problem for the 5G/Beyond-5G evolution. DSA allows unused frequency resources that are primarily allocated to existing wireless systems (hereafter referred to as primary system (PS)) to be utilized by other wireless systems (hereafter referred to as secondary system (SS)) in a secondary use manner. In DSA, the SS have to coexist with the PS in the same frequency band without causing any harmful interference to the PS (hereafter this technology is referred to as incumbent protection). To make the coexistence into practice, an interference coordination system, also known as a spectrum management/access system, geolocation database, and so on, is regarded as a core component of DSA. DSA and its relevant legislation, standardization and proof-of-concept activities have been conducted worldwide [1, 2, 3, 4].

In Japan, the Ministry of Internal Affairs and Communications (MIC) has formulated an action plan for frequency reallocation [4]. In the plan, the MIC mentions DSA for the $5 \mathrm{G}$ systems in a $26 \mathrm{GHz}$ band, which is primarily assigned to fixed wireless access (FWA). However, since the SSs are based on 4G LTE in the ongoing studies and standardizations, conventional incumbent protection methods cannot be adopted to the 5G SS. For example, the conventional methods do not take any kind of beamforming characteristics, which is a major feature of $5 \mathrm{G}$, of the SS into account.

To explore a more advanced DSA towards 5G/Beyond-5G, we propose a new incumbent protection method in this letter. Our proposed method considers the threedimensional (3D) aspects of the $5 \mathrm{G}$ beamforming characteristics. The proposed method further introduces a concept of area-based protection to satisfy protection criteria for a PS with inaccurate location information [5]. In the followings, we explain an algorithm of the proposed method. Then we show some computer simulation results for deploying a $5 \mathrm{G}$ based SS with the 3D beamforming in a millimeter-wave band which is primarily assigned to a fixed wireless access (FWA) based PS. The simulation results demonstrate that maximum allowable transmission powers of the SS can be increased by the proposed method considering the 3D beamforming aspects and the area-based protection. 


\section{Proposed incumbent protection method}

\subsection{Area-based protection}

In DSA, an interference coordination system carries out the incumbent protection algorithms by using operational information of PS and SS. We proposed a concept of area-based protection for practical DSA scenarios in which the interference coordination system could not obtain accurate location information from the PS [5]. The area-based protection introduces a protection area (PA) and a grid of protection points (PPs) in the PA. The area-based protection assumes that a radio station of the PS is virtually located at each PP. Then SS's transmission parameters, such as transmission power and frequency channels, are adjusted to make potential interferences from the SS to the PS below an acceptable level at all the PPs. In this letter, we combine the area-based protection into the transmission power calculation considering the $3 \mathrm{D}$ beamforming aspects.

\subsection{Allowable transmission power calculation}

Our proposed method aims at two things: (a) to calculate allowable transmission power per beam pattern per SS radio station (SSS), and (b) to increase allowable transmission powers of beam patterns which will not cause any harmful interference to the PS and surrounding PPs in the PA.

Fig. 1 illustrates a geolocation model when calculating the allowable maximum transmission power of the SS. In this subsection, for simplicity, let us assume a pair of two PS radio stations (PSSs) in a PA, and an SSS $^{1}$ such as an SS base station. Also we assume that a total $K$ beam patterns are available at the SSS. $\theta_{Z o D, p}$ and $\phi_{A o D, p}$ represent zenith and azimuth angles of departure ( $\mathrm{ZoD}$ and $\left.\mathrm{AoD}\right)$ from the SSS to a PP $p$ in the PA. $\theta_{Z o A, p}$ and $\phi_{A o A, p}$ represent zenith and azimuth angles of arrival (ZoA and AoA) at the PP.

When the SSS uses the $k$-th beam pattern, a potential interference power to the PP $I_{k, p}(\mathrm{dBm})$ can be expressed as follows:

$$
I_{k, p}=P_{S S}+G_{S S, k}\left(\theta_{Z o D, p}, \phi_{A o D, p}\right)-L_{S S \rightarrow p}+G_{P S}\left(\theta_{Z o A, p}, \phi_{A o A, p}\right),
$$

where $P_{S S}(\mathrm{dBm})$ and $L_{S S \rightarrow p}(\mathrm{~dB})$ are a transmission power of the SSS and a propagation loss from the SSS to the PP, respectively. Also, $G_{S S, k}\left(\theta_{Z o D, p}, \phi_{A o D, p}\right)$

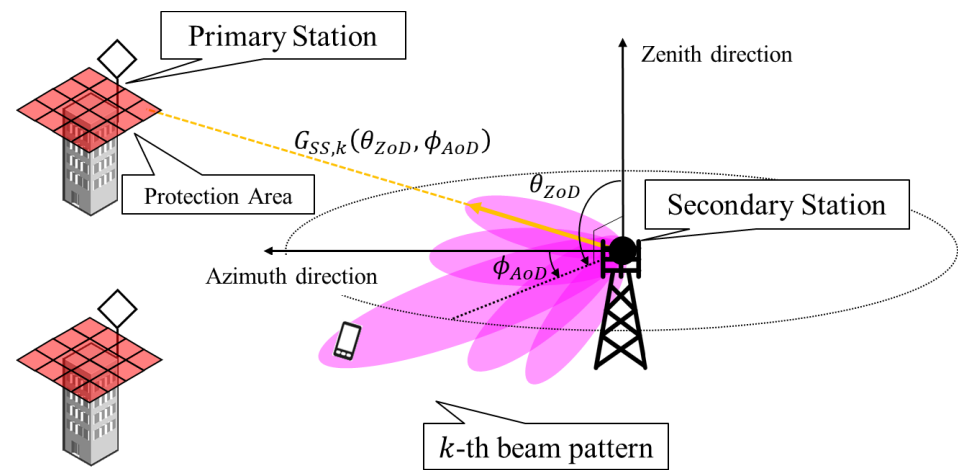

Fig. 1. Geolocation model for the proposed method.

๑) IEICE 2021

DOI: 10.1587/comex.2021ETL0037 Received February 16, 2021 Accepted March 10, 2021
${ }^{1}$ Note that our proposed method can be extended to cases of multiple SSS and aggregate interference calculation, e.g. based on [5]. 
$(\mathrm{dB})$ is the $k$-th beam pattern's gain of the SSS in the direction of $\theta_{Z o D, p}$ and $\phi_{A o D, p}$. Similarly, $G_{P S}\left(\theta_{Z o A, p}, \phi_{A o A, p}\right)(\mathrm{dB})$ is an antenna gain of the PSS in the direction of $\theta_{Z o A, p}$ and $\phi_{A o A, p}$. To keep the potential interference to the PSS at the PP below an acceptable interference power $I_{\text {Threshold }}(\mathrm{dBm})$, the allowable transmission power level of the SSS's $k$-th beam pattern $P_{S S, k, p}$ can be calculated as follows:

$$
P_{S S, k, p}=I_{\text {Threshold }}-G_{S S, k}\left(\theta_{Z o D, p}, \phi_{A o D, p}\right)+L_{S S \rightarrow p}-G_{P S}\left(\theta_{Z o A, p}, \phi_{A o A, p}\right) \text {. }
$$

Since the proposed method considers the area-based protection, each beam pattern of the SSS shall satisfy the condition of the acceptable interference power for all the PPs in the PA. Then the maximum allowable transmission power of the $k$-th beam pattern should be finally set as follows:

$$
P_{S S, k}=\min \left\{P_{S S, k, p}\right\}, \quad p \in P A .
$$

\section{Performance evaluation}

\subsection{Simulation parameters}

In this letter, we evaluate the proposed method by computer simulation and compare it with a conventional method [6]. The conventional method has been adopted in the CBRS (Citizens Broadband Radio Service) for 4G-based SSs, and therefore it does not consider any kind of beamforming characteristics of 5G-based SSs. In the simulation, we assumed a pair of two FWA radio stations as PSSs and a $5 \mathrm{G}$ base station having three sectors as an SSS in a $26 \mathrm{GHz}$ frequency band. Table I [ $7,8,9]$ shows details of simulation parameters.

\subsection{Comparison of maximum allowable EIRP}

Fig. 2 compares the maximum allowable EIRPs (Equivalent Isotropic Radiated Powers) of the SS calculated by the conventional and proposed methods with the different PA sizes. In Fig. 2 we also plot EIRP without PS as a reference. In

\begin{tabular}{|c|c|c|}
\hline \multicolumn{3}{|c|}{ Deployment parameters } \\
\hline Number of PSSs & \multicolumn{2}{|c|}{2} \\
\hline Number of SSSs & \multicolumn{2}{|c|}{1 base station with 3 sectors } \\
\hline PA size (square) & \multicolumn{2}{|c|}{$0,50 \mathrm{~m} \times 50 \mathrm{~m}, 100 \mathrm{~m} \times 100 \mathrm{~m}, 200 \mathrm{~m} \times 200 \mathrm{~m}$} \\
\hline PP interval within PA & \multicolumn{2}{|c|}{$10 \mathrm{~m}$} \\
\hline Distance between PSS and SSS & \multicolumn{2}{|l|}{$1118 \mathrm{~m}$} \\
\hline \multicolumn{3}{|c|}{ Radio parameters } \\
\hline System & PSS (FWA) & SSS (5G) \\
\hline Center frequency & \multicolumn{2}{|c|}{$26.5 \mathrm{GHz}$} \\
\hline Bandwidth & $58.5 \mathrm{MHz}$ & $100 \mathrm{MHz}$ \\
\hline Maximum transmission power & - & $25 \mathrm{dBm}$ \\
\hline Antenna model & ITU-R F.699 & $\begin{array}{l}\text { 3GPP TR38.901, } \\
16 \times 16 \text { elements, } \\
5 \text { dBi element gain }\end{array}$ \\
\hline Antenna height & $40 \mathrm{~m}$ & $\begin{array}{l}\text { Base station: } 16 \mathrm{~m} \text {, } \\
\text { Users: } 1.5 \mathrm{~m}\end{array}$ \\
\hline Acceptable interference level & $-96.8 \mathrm{dBm}$ & - \\
\hline Radio propagation model & \multicolumn{2}{|c|}{ 3GPP TR38.901 } \\
\hline
\end{tabular}

Table I. Simulation parameters $[7,8,9]$. 


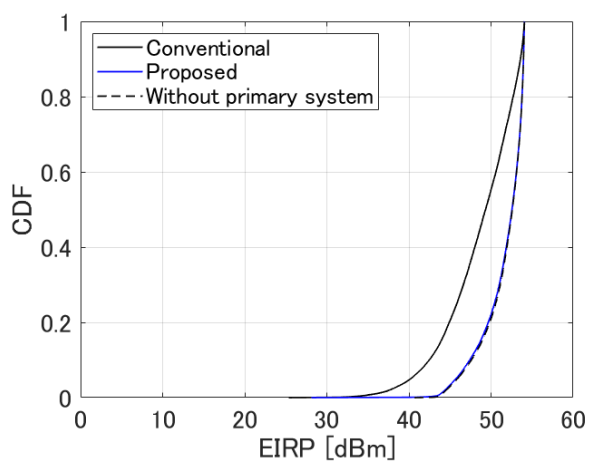

(a) $\mathrm{PA}$ size $=0$ (i.e. point protection).

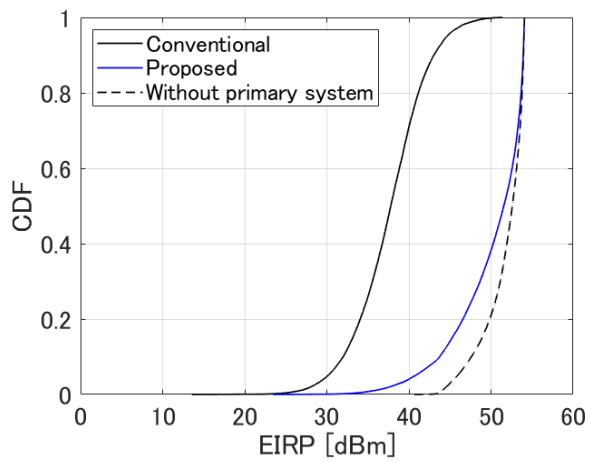

(c) PA size $=100 \mathrm{~m} \times 100 \mathrm{~m}$.

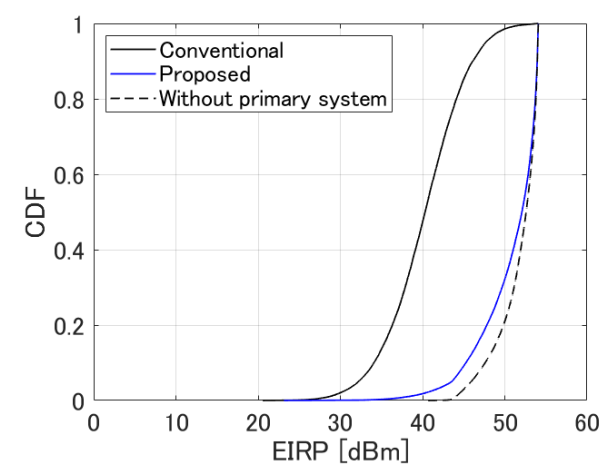

(b) PA size $=50 \mathrm{~m} \times 50 \mathrm{~m}$.

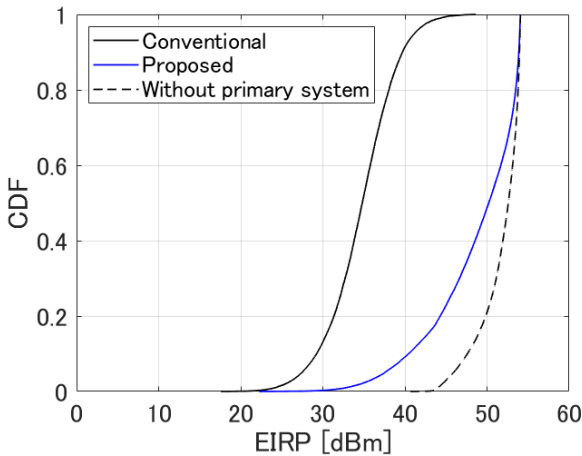

(d) PA size $=200 \mathrm{~m} \times 200 \mathrm{~m}$.

Fig. 2. Comparison of the allowable EIRP at SSS.

the conventional method, a worst-case allowable transmission power is applied for all beam patterns in common. Therefore, the conventional method's EIRP is decreasing especially when the PA size becomes large. On the other hand, the proposed method dramatically increases the maximum allowable EIRP thanks to the interference calculation per beam pattern. The proposed method shows more advantage compared to the conventional method when the PA size becomes large. In Fig. 2(d), for example, the proposed method increases the EIRP by $15 \mathrm{~dB}$ at 50 percentile $\mathrm{CDF}$, and by $10 \mathrm{~dB}$ at 5-percentile CDF. Note that the proposed method achieves almost the same EIRP performance without PS when the PA size is zero (i.e. point-based protection). Even in the other situations of the PA sizes, losses of the allowable EIRP were relatively small.

\section{Conclusion}

In this letter, we proposed an area-based incumbent protection method considering the SSs' 3D beamforming aspects in DSA. The simulation results showed that the proposed method can make use of the benefits of the beamforming in improving the maximum allowable transmission powers of the SSs in DSA.

\section{Acknowledgments}

This research is supported by the Ministry of Internal Affairs and Communications in Japan (JPJ000254). 